\title{
Group A Streptococcal Carriage in Children with ADHD: Antibiotic Resistance and Associated ASO Levels
}

\author{
Eunice Swarna Jacob, S. Matheskumar* and Rishab Bharadwaj \\ Department of Microbiology, Thanjavur Medical College, Chennai, India \\ *Corresponding author
}

\begin{tabular}{|c|}
\hline Keywords \\
\hline $\begin{array}{l}\text { GAS - Resistance- } \\
\text { Macrolides - ASO }\end{array}$ \\
\hline Article Info \\
\hline $\begin{array}{l}\text { Accepted: } \\
\text { 07 February } 2019 \\
\text { Available Online: } \\
10 \text { March } 2019\end{array}$ \\
\hline
\end{tabular}

\section{A B S T R A C T}

Acute pharyngitis is a common illness of childhood most frequently associated with Group A Streptococcus $(G A S)$ and carrier state quiet often remains undiagnosed in children with attention deficit hyperactivity disorder. This prospective study was undertaken to assess the burden of GAS in children with attention deficit hyperactivity disorder attending pediatric clinics in a tertiary care centre at Chennai. Throat swabs were obtained for culture from 50 ADHD children aged $5-15$ years with symptoms of sore throat and 50 from apparently asymptomatic children with ADHD. Beta hemolytic streptococcal isolates were isolated and serogrouped and subjected to antibiotic susceptibility testing as per CLSI standards. Blood samples were collected from all 100 children to determine elevated ASO levels. 24 children of the symptomatic study group were positive for beta hemolytic streptococci. Asymptomatic carriage of GAS was 28\%. Antibiotic resistance to erythromycin, azithromycin and ofloxacin was high. All strains were sensitive to Penicillin. Nineteen children had elevated ASO titre levels. Periodic surveillance is mandatory to prevent the spread of GAS carriage in children with ADHD. Multidrug resistant phenotypes are emerging. Regular monitoring of drug resistance will serve as a valuable tool in preventing periodic outbreak in the community.

\section{Introduction}

Group A streptococcus is the most common bacterial cause of acute pharyngitis accounting for about $15-30 \%$ of cases in children and 5-10\% in adults. Pharyngitis due to GAS is primarily a disorder of children 5 15 years of age (19). Streptococcal carriage is defined as the recovery of GAS from the upper respiratory tract in the absence of any evidence of acute infection ${ }^{5}$. GAS colonizes the mucous membrane of throat and skin causing a panorama of infections like pharyngitis, impetigo, scarlet fever and also responsible for life threatening complications like toxic shock syndrome. In recent years it has gained popularity as 'flesh eating bacteria'. GAS has a unique role in the non suppurative sequelae which includes Acute Rheumatic fever (ARF), Acute glomerulonephritis (AGN), Reactive arthritis and Pediatric Autoimmune diseases associated with Streptococcal infections (PANDAS) $)^{4}$ Attention deficit hyperactivity 
disorder is a neurological condition defined by consistent pattern of inattention or hyperactive impulsivity that interferes with the daily functioning impacting children and adults, boys and girls and people of all backgrounds.

Rheumatic heart disease (RHD) remains the major cause of cardiovascular morbidity and mortality in India though it has declined in many parts of the world. The RHD incidence in India varies from $0.2-0.5 / 1000 /$ year in schoolchildren 5-15 years of age. The younger age of onset of RHD is more severe and rapidly progressive. ${ }^{6}$

Despite the widespread use of Penicillin in treating GAS infections, no resistance has been reported. Macrolides and quinolones are suitable alternatives indicated for individuals who are non tolerant to penicillin. There is an alarming increase in resistance to penicillin and other antibiotics and has been reported worldwide.

This preliminary study was thus undertaken to investigate the rate of pharyngeal colonization of GAS, drug susceptibility and associated ASO levels among children with attention deficit hyperactivity disorder.

\section{Materials and Methods}

The study group included ADHD children between 5 - 15 years attending pediatric clinics in a tertiary care hospital. Throat swabs were collected from 50 children with fever, sore throat, difficulty in swallowing and swollen inflamed tonsils with or without follicles and 50 throat swabs were collected from asymptomatic ADHD children. Children who have been previously treated for sore throat or any other infection during the past week were excluded from the study. Blood samples were collected from all children of the study group to determine the ASO levels.
The swabs were transported to the laboratory, inoculated onto 5\% blood agar plates incubated overnight in an atmosphere of 5$10 \% \mathrm{CO}_{2}$. Colonies showing beta hemolysis, sensitive to $0.04 \mathrm{U}$ bacitracin, gram positive cocci in chains, catalase negative were then serogrouped using commercially available specific antisera. The study was conducted between July and September 2018

GAS isolates were then screened for susceptibility to Penicillin (10U), Erythromycin $(15 \mu \mathrm{g})$, Azithromycin $(15 \mu \mathrm{g})$, Clindamycin $(2 \mu \mathrm{g})$, Levofloxacin $(5 \mu \mathrm{g})$, CoTrimoxazole (Trimethoprim/ Sulphamethoxazole) (1.25/23-75 $\mu$ g) by Kirby Bauer disc diffusion method on Mueller Hinton agar supplemented with 5\% blood. The diameter of the zone of inhibition was measured and interpreted as per Clinical Laboratory Standards Institute (CLSI) guidelines.

Blood samples were centrifuged, serum separated and ASO test was done by latex agglutination (Beacon diagnostics) as per the manufacture's instructions.

\section{Results and Discussion}

Of the 100 swabs cultured 36 GAS isolates were obtained, 24(48\%) from symptomatic and $9(18 \%)$ from asymptomatic children were isolated. 22 GAS isolates were obtained in the age group of 9-12years. Gender distribution was not significant. All isolates were sensitive to Penicillin followed by Clindamycin $81 \%$ (29/36). Erythromycin and Azithromycin resistance was encountered in $75 \%(27 / 36)$ and $52 \%$ (4/36) of GAS strains. 20 (55.5\%) isolates showed intermediate susceptibility to macrolides. Reduced susceptibility to Levofloxacin was observed in $47 \%$ (17/36) of the GAS strains. $95 \%$ of the strains were resistant to Cotrimoxazole. 
Among the 100 serum samples tested for ASO, 19 were found to be positive. 12 (24\%) was observed in the symptomatic group and $7(14 \%)$ in the asymptomatic group. The titre values were found to be between 200IU $800 \mathrm{IU} / \mathrm{ml}$. The symptomatic group who were both culture and ASO positive (5/12) showed titre value of $800 \mathrm{IU} / \mathrm{ml}$ whereas symptomatic culture negative but ASO positive (7/12) had a titre value of $200-400 \mathrm{IU} / \mathrm{ml}$. The asymptomatic group showed a titre of 200IU/ml.

Pharyngeal carriage rates among school children vary with socioeconomic status, geographic location and season the year. The children in the study group belonged to lower socioeconomic status with attention deficit hyperactivity disorder where overcrowding and intrafamilial spread is a problem. ${ }^{8}$ Chennai has a hot and humid tropical climate with intermittent monsoon in the months of July and August which are favorable factors contributing to the spread of the infection and a high prevalence rate of $46 \%$ in symptomatic and $26 \%$ in asymptomatic children in our study.

The global burden of GAS is estimated to be 616 million pharyngitis cases per year ${ }^{2}$. Epidemiological surveys in Pittsburg, Korea and Melboune have reported prevalence rates of $15.5 \%, 16.9 \%$ and $16 \%$. Previous studies have reported GAS carriage rate to be $2.3 \%$ and $7.8 \%$ in rural population of South India. One study in Chennai have reported a rate of $13.6 \%$ in school children. ${ }^{7}$

\section{Acknowledgement}

We acknowledge the Indian Council of Medical Research, New Delhi, India for funding this study form of a short term research studentship to the author Rishab Bharadwaj.

\section{References}

1. Koshi G, Benjamin V: Surveillance of Streptococcal Infections in a South Indian Community-A Pilot survey; Indian J Med Res, Sep 1977; Vol.66, 379388;

2. Carapetis JR, Steer AC, Mulholland EK, Weber M. The global burden of group a streptococcal diseases. Lancet Infect Dis 2005; 5: 685-94

3. Menon T, Shanmugasundaram S, Kumar MP, Kumar CPG: Group A Streptococcal infections of the pharynx in a rural population in S. India; Indian J Med Res, 2004; 119 Suppl: 171-3;

4. Cunningham MW: Pathogenesis of Group A Streptococcal Infections; Clini. Microbiol. Rev, July 2000; 470-511, Vol. 13, No. 3;

5. Pichechero ME, Casey JR: Defining and dealing with carriers of group A Streptococci; Contemporary Pediatrics, 2003; 1: 46.

6. Anil G, Rajesh Vijayvergiya, Shyan T Thingam: Burden of Rheumatic and Congenital Heart Disease in India: Lowest estimate based on the 2001 Census; Indian Heart Journal, Jan-Feb 2001.

7. Charmaine AC Llyod, Swarna Jacob and Thangam Menon et al., Pharyngeal carriage of group A Streptococci in school children in Chennai; Indian J Med Res, August 2006; 124, 195-198;

8. Kaplan E: The group A streptococcal upper respiratory tract carrier state: An enigma; J. Pediatrics, Sep 1980; Vol. 97, No 3, 337-345;

9. Martin JM, Green M, et al.,: Group A Streptococci among school-aged children: clinical characteristics and the carrier State; Pediatrics, 114; 2004

10. Margaret HD, Susan R, et al.,: The burden of group A streptococcal pharyngitis in Melbourne Families; 
Indian JMed Res, 144-147, 119 (Suppl); May 2004

11. Kim S, Yong Lee N: Epidemiology and antibiotic resistance of group A
Streptococci isolated from healthy schoolchildren in Korea; J. Antimicrob. Chemother, 54, 447-450; 2004.

\section{How to cite this article:}

Eunice Swarna Jacob, S. Matheskumar and Rishab Bharadwaj. 2019. Group A Streptococcal Carriage in Children with ADHD: Antibiotic Resistance and Associated ASO Levels. Int.J.Curr.Microbiol.App.Sci. 8(03): 472-475. doi: https://doi.org/10.20546/ijcmas.2019.803.059 